\title{
Local methacholine but not bradykinin potentiates insulin-mediated glucose uptake in muscle in vivo by augmenting capillary recruitment
}

\author{
H. Mahajan ${ }^{1}$ S. M. Richards ${ }^{1}$ S. Rattigan ${ }^{1}$ M. G. Clark ${ }^{1}$ \\ ${ }^{1}$ Biochemistry, School of Medicine, University of Tasmania, Hobart, Tasmania, Australia
}

\begin{abstract}
Aims/hypothesis. Insulin has nitric-oxide-dependent vasodilatory effects in muscle, including capillary recruitment, that enhance access for itself and glucose. However, nitric-oxide-dependent vasodilators other than methacholine do not enhance insulin action. Our hypothesis is that methacholine, unlike bradykinin, enhances insulin-mediated glucose uptake in muscle by augmenting capillary recruitment.

Methods. Local infusion of either methacholine or bradykinin into one leg of the anaesthetised rat was made during physiological insulin $\left(3 \mathrm{mU} \cdot \mathrm{kg}^{-1} \cdot \mathrm{min}^{-1}\right)$ infusion under euglycaemic conditions and without affecting systemic blood pressure. Whole-body glucose infusion was determined, as was femoral blood flow, 2-deoxyglucose uptake into calf muscles and the metabolism of infused 1-methylxanthine, a measure of capillary recruitment for each leg.

Results. Methacholine alone $\left(0.3 \mu \mathrm{mol} \cdot \mathrm{l}^{-1}\right)$ increased femoral arterial blood flow, increased capillary recruitment but had no effect on 2-deoxyglucose uptake
\end{abstract}

of the test leg relative to the contra-lateral control leg. Insulin alone (systemically) required a glucose infusion rate of $8.7 \mathrm{mg} \cdot \mathrm{kg}^{-1} \cdot \mathrm{min}^{-1}$ to maintain euglycaemia, increased 2-deoxyglucose uptake and capillary recruitment, but was without effect on femoral blood flow in either leg. Local methacholine with systemic insulin infusion increased femoral blood flow, 2-deoxyglucose uptake and capillary recruitment in the test leg only. Bradykinin $\left(0.07 \mu \mathrm{mol} \cdot \mathrm{l}^{-1}\right)$, alone or with insulin, administered in a manner that increased femoral blood flow similarly to methacholine, did not affect 2-deoxyglucose uptake or capillary recruitment. Conclusions/interpretation. Methacholine but not bradykinin enhances insulin-mediated muscle glucose uptake in vivo. We conclude that methacholine acts at specific sites in the vasculature of muscle to stimulate capillary recruitment and thereby enhance insulin access.

Keywords Global vasodilatation in muscle · Insulin and glucose delivery to muscle $\cdot$ Muscle blood flow Specific regional vasodilatation in muscle.
Received: 7 May 2004 / Accepted: 18 July 2004

Published online: 16 December 2004

(C) Springer-Verlag 2004

M. G. Clark (๘)

Biochemistry, School of Medicine, University of Tasmania,

Private Bag 58, Hobart, Tasmania, Australia, 7001

E-mail: Michael.Clark@utas.edu.au

Tel.: +61-3-62262672, Fax: +61-3-62262703

Abbreviations: $\mathrm{BP}$, mean arterial blood pressure . 2DG, 2-deoxyglucose - FBF, femoral arterial blood flow . GIR, whole-body glucose infusion rate .

1-MX, 1-methylxanthine · NO, nitric oxide ·

$0 R^{\prime} \mathrm{g}$, muscle $2 \mathrm{DG}$ uptake $\cdot \mathrm{VR}$, vascular resistance

\section{Introduction}

There is evidence that a rate-limiting step for insulinmediated glucose uptake by muscle in vivo is at the level of insulin and glucose delivery [1]. In addition, there is circumstantial evidence that this step may become even further limiting in insulin-resistant states $[1,2]$. Under normal conditions in vivo insulin exerts a number of haemodynamic effects within skeletal muscle including an increase in bulk blood flow (usually determined as limb blood flow [3]) and capillary recruitment [4]. While each of these is nitric-oxide (NO)-dependent $[5,6,7]$ and has been proposed to en- 
hance delivery of glucose and insulin to the muscle cells [8], recent evidence shows that capillary recruitment correlates more closely than bulk flow with glucose uptake [9]. For physiological insulin, moreover, capillary recruitment is an early [10] and highly sensitive process [11]. In contrast, bulk flow is slow to respond at physiological insulin levels and only rapid when doses are raised above physiological levels [12]. When vasodilators such as bradykinin [13], nitroprusside [14], or adenosine [15] are infused, bulk flow is markedly augmented without an effect on insulin action. These results suggest that bulk blood flow changes are not necessary for insulin's metabolic actions in muscle. One exception is the vasodilator methacholine, which increases total flow, and has been reported by two groups to enhance insulin action. Thus in 1994 methacholine was reported to augment insulin-mediated glucose uptake across the leg of young healthy subjects [3]. Similarly, five years later methacholine was found to increase forearm glucose uptake of hypertensive insulin-resistant subjects [16]. Thus it is puzzling why various vasoactive agents that act via NO-dependent vasodilatation have such different metabolic outcomes when the haemodynamic actions of insulin are also NO-dependent. It has been proposed [3] that the difference in vasodilator metabolic action may relate to the specific sites affected within the vasculature. Accordingly, in the present study and using a novel approach of local infusion in the rat leg in vivo, we compare the metabolic effects of methacholine and bradykinin on physiological insulin in terms of macro- and micro vascular haemodynamic changes.

\section{Materials and methods}

Animals. All procedures adopted and experiments undertaken were approved by the University of Tasmania Animal Ethics Committee. Male hooded Wistar rats weighing 281 $\pm 6 \mathrm{~g}$ were bred in house at the University of Tasmania Animal House (Hobart, Tasmania, Australia) and raised on a commercial diet (Pivot, Launceston, Australia) containing 21.4\% protein, $4.6 \%$ lipid, $68 \%$ carbohydrate, and $6 \%$ crude fibre with added vitamins and minerals, together with free access to water. Until the day of surgery, rats were housed at a constant temperature of $21 \pm 1^{\circ} \mathrm{C}$ with a $12-\mathrm{h} / 12$-h light/dark cycle. Food and water were freely available until the animals were anaesthetised.

Surgical preparation. The intention was to conduct a hyperinsulinaemic-euglycaemic clamp in rats so that the effects of a local muscle infusion of the vasodilators methacholine and bradykinin could be assessed in the absence of systemic perturbations. Particular focus was on effects imposed on the haemodynamic effects of insulin. Details for the clamp were essentially as described previously [4] with some modifications; a schematic drawing showing the positioning of cannulae and flow probes is given in Figure 1a. The rats were anaesthetised using Nembutal (50 $\mathrm{mg} \cdot \mathrm{kg}^{-1}$ body weight) and had polyethylene cannulas (PE-50; Intramedic, Becton Dickinson, Parsippany, N.J., USA) surgically implanted into the carotid artery for arterial sampling and measurement of blood pressure (pressure transducer Transpac IV; Abbott Critical Care Systems, Morgan Hill, Calif., USA) and into both jugular veins for continuous administration of anaesthetic and other intravenous infusions. A tracheotomy tube was inserted, and the animal was allowed to spontaneously breathe room air throughout the course of the experiment. Small incisions $(1.5 \mathrm{~cm})$ were made in the skin overlaying the femoral vessels of both legs, and the femoral artery was separated from the femoral vein and saphenous nerve. In one leg (test leg), the epigastric artery was cannulated and an ultrasonic flow probe (VB series $0.5 \mathrm{~mm}$; Transonic Systems, Ithaca, N.Y., USA) was positioned around the femoral artery just distal to the rectus abdominis muscle prior to the point where the epigastric artery leaves the femoral artery (Fig. 1a). In the contralateral leg (control leg) the epigastric vessels were ligated and a second ultrasonic flow probe was positioned around the femoral artery as above. The probes were connected to flow meters (T106 ultrasonic volume flow meter; Transonic Systems). These were in turn interfaced with an IBM-compatible PC computer, which received the data (at a sampling frequency of $100 \mathrm{~Hz}$ ) for femoral blood flow, heart rate, and blood pressure using WINDAQ data acquisition software (DATAQ Instruments, Akron, Ohio, USA). The animals were maintained under anaesthesia for the duration of the experiment using a continual infusion of Nembutal $\left(0.6 \mathrm{mg} \cdot \mathrm{kg}^{-1}\right.$. $\mathrm{min}^{-1}$ ) via the left jugular cannula. The body temperature was maintained using a water-jacketed platform and a heating lamp positioned above the rat.

Euglycaemic-hyperinsulinaemic clamps. Once the surgery was completed, a 45 to 60 -min equilibration period was allowed so leg blood flow and blood pressure could become stable and constant. Rats were then subjected to the protocol (Fig. 1b), during which they were infused systemically with saline or insulin (as a euglycaemic insulin clamp with $3 \mathrm{mU} \cdot \mathrm{kg}^{-1} \cdot \mathrm{min}^{-1}$ ) (Humulin R; Eli Lilly, Indianapolis, Ind., USA) from $t=0$ for $2 \mathrm{~h}$. This rate of insulin infusion was chosen as it has little or no effect on femoral arterial blood flow (FBF) [11]. Local infusion of methacholine or bradykinin into the epigastric artery of the test leg was made for the last $45 \mathrm{~min}$. Preliminary experiments were conducted to determine which infusion rate of methacholine or bradykinin would produce maximal increase in FBF without effects on mean arterial blood pressure or heart rate. Some experiments involved systemic infusions of methacholine intra-arterially for the final $20 \mathrm{~min}$ of either saline $(n=5)$ or insulin clamp $\left(3 \mathrm{mU} \cdot \mathrm{kg}^{-1} \cdot \mathrm{min}^{-1} ; n=5\right)$.

Blood samples. Arterial samples were taken at the times indicated (Fig. 1) for blood glucose (all) and insulin (-5 and 120min) measurements. The femoral vein of each leg was used for venous sampling, using a $29 \mathrm{G}$ insulin syringe (Becton Dickinson). Duplicate venous samples $(300 \mu \mathrm{l})$ were taken only on completion of the experiment (total time $120 \mathrm{~min}$ ) to prevent alteration of the blood flow from the hindlimb due to sampling, and to minimise the effects of blood loss. The blood samples were placed on ice, immediately centrifuged and the plasma stored at $-20^{\circ} \mathrm{C}$ until assayed. The total blood volume withdrawn from the animals before the final arterial and venous samples did not exceed $1.5 \mathrm{ml}$ and was easily compensated for by the volume of fluid infused.

Capillary recruitment. Capillary recruitment was determined by measuring the metabolism of infused 1-methylxanthine (1-MX), a substrate targeted for xanthine oxidase, which in the hindlimb muscle is located predominantly in capillary endothelium [17]. We infused 1-MX (Sigma Aldrich, St. Louis, Mo., USA) at a constant rate $\left(0.5 \mathrm{mg} \cdot \mathrm{kg}^{-1} \cdot \mathrm{min}^{-1}\right)$ to maintain a steady-state arte- 

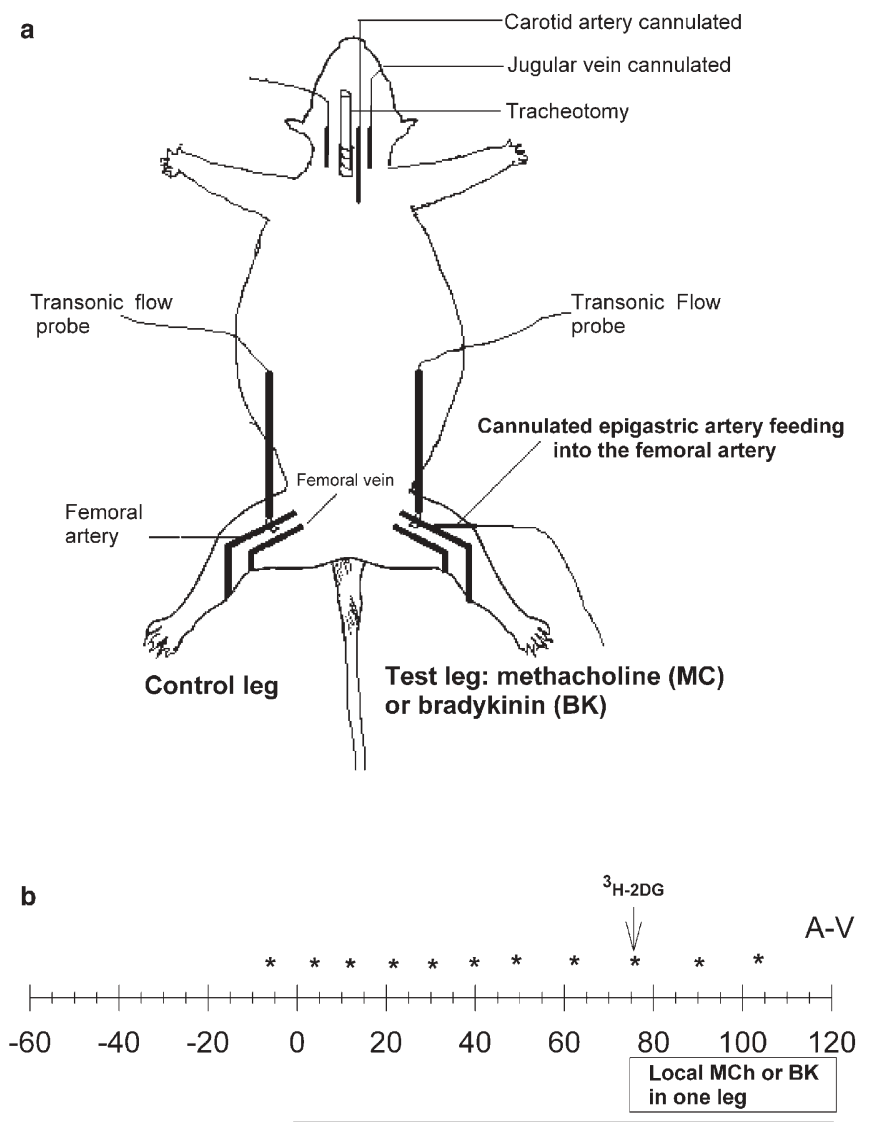

Systemic insulin $\left(3 \mathrm{mU} \cdot \mathrm{kg}^{-1} \cdot \mathrm{min}^{-1}\right)$ or saline

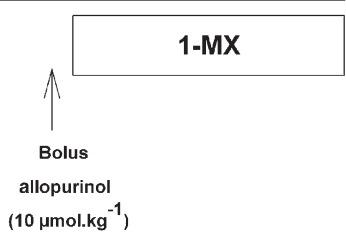

Fig. 1 a. Schematic drawing showing the positioning of cannulae and flow probes. Cannulae were inserted in a carotid artery (BP measurements), each jugular vein and one epigastric artery (test leg; methacholine [MC] or bradykinin [BK] infusions). Other infusions were anaesthetic (jugular vein), insulin/saline (jugular vein), and glucose (jugular vein). Double infusions into a jugular vein were achieved by a four-way tap. Transonic flow probes were positioned around each femoral artery. b. Study design involved the euglycaemic clamp at $3 \mathrm{mU} \cdot \mathrm{kg}^{-1}$. $\mathrm{min}^{-1}$ insulin or saline commencing at time $=0 \mathrm{~min}$ and continuing for $120 \mathrm{~min}$. MC $\left(1 \mu \mathrm{g} \cdot \mathrm{kg}^{-1} \cdot \mathrm{min}^{-1}\right)$ or BK $\left(2 \mu \mathrm{g} \cdot \mathrm{kg}^{-1}\right.$. $\min ^{-1}$ ) was infused into the epigastric artery of the test leg for the last $45 \mathrm{~min}$. Systemic venous infusions are indicated by the bars. Duplicate arterial and femoral venous plasma samples (indicated by A-V) were collected from each hind leg (test and contralateral control) at $120 \mathrm{~min}$, for HPLC analysis, and plasma glucose determinations. Bolus systemic injections of allopurinol or 2-DG were made as indicated (arrows). Arterial samples for glucose (all) and insulin (-5 and $120 \mathrm{~min}$ ) determinations are indicated by *. Muscle samples were taken at $120 \mathrm{~min}$ for 2-DG. The numbers of animals in each group were: $\mathrm{MC}, n=5 ; \mathrm{MC}+$ insulin, $n=6 ; \mathrm{BK}, n=6 ; \mathrm{BK}+$ insulin, $n=6$ rial level of approximately $20 \mu \mathrm{mol} / \mathrm{l}$. As noted previously [4, 9], this was achieved by partly inhibiting the activity of xanthine oxidase with allopurinol [18] $\left(10 \mu \mathrm{mol} \cdot \mathrm{kg}^{-1}\right)$, administered as a bolus dose 5 minutes prior to commencing the 1-MX infusion (Fig. 1). This not only allowed constant arterial concentrations of 1-MX to be maintained throughout the experiment, but lowered the $K_{\mathrm{m}}$ of the enzyme sufficiently so that $20 \mu \mathrm{mol} \cdot \mathrm{l}^{-1} 1-\mathrm{MX}$ was well above saturation (unpublished observations).

Plasma $(20 \mu \mathrm{l})$ from arterial and leg venous blood samples taken at the end of the experiment was mixed with $8 \mu \mathrm{l}$ of $0.42 \mathrm{~mol} \cdot \mathrm{l}^{-1}$ perchloric acid and centrifuged for $10 \mathrm{~min}$. The supernatant was used to determine 1-MX, allopurinol and oxypurinol concentrations by reverse-phase HPLC as previously described [4]. Capillary recruitment, expressed as 1-MX metabolism was calculated from the difference between arterial and venous plasma 1-MX concentrations and multiplied by femoral blood flow. The concentration in plasma was corrected for the volume accessible to 1-MX, determined from plasma concentrations obtained after additions of standard 1-MX to whole rat blood to be $87.1 \%$. The rate of 1 -MX metabolism across the hindlimb (expressed as nmol. $\mathrm{min}^{-1}$ ) reflects conversion by capillary endothelial xanthine oxidase to the sole product, 1-methyl urate [19].

Muscle glucose uptake. At 45 min prior to the completion of each experiment (Fig. 1b), a $1.85 \mathrm{MBq}$ bolus of 2-deoxy-D$\left[2,6-{ }^{3} \mathrm{H}\right]$ glucose $(2 \mathrm{DG})\left(\right.$ specific activity $=1.92 \mathrm{TBq} \cdot \mathrm{mmol}^{-1}$ ) (Amersham Life Science, Castle Hill, NSW, Australia) in saline was administered. Plasma samples $(25 \mu \mathrm{l})$ were collected at $5,10,15,30$ and $45 \mathrm{~min}$ after $2 \mathrm{DG}$ to determine plasma clearance of the radioactivity. At the conclusion of the experiment, the soleus, plantaris, gastrocnemius white, gastrocnemius red, EDL and tibialis muscles were removed, clampfrozen in liquid nitrogen and stored at $-20^{\circ} \mathrm{C}$.

Individual frozen muscles were ground under liquid nitrogen and homogenised with 10 volumes of water using an Ultra Turrax. Free and phosphorylated $\left[{ }^{3} \mathrm{H}\right] 2 \mathrm{DG}$ were separated by ion exchange chromatography using an anion exchange resin (AG1-X8) [20, 21]. Biodegradable Counting Scintillant-BCA (Amersham) was added to each radioactive sample and radioactivity determined using a scintillation counter (LS3801; Beckman Instruments, Fullerton, Calif., USA). From this measurement and a knowledge of plasma glucose and the time course of plasma 2DG disappearance, muscle 2DG uptake $\left(\mathrm{R}^{\prime} \mathrm{g}\right)$, which reflects glucose uptake into the muscle, was calculated as previously described by others $[20,21]$.

Other assays. A glucose analyser (2300 Stat plus; Yellow Springs Instruments, Yellow Springs, Ohio, USA) was used to determine whole blood glucose (by the glucose oxidase method) during the insulin clamp. A blood sample of $25 \mu \mathrm{l}$ was required for each measurement. Insulin assays were conducted on arterial plasma samples taken at $-5 \mathrm{~min}$ (pre-insulin) and at 120 min (post-insulin) using an ELISA method (Mercodia, Uppsala, Sweden).

Expression of results. All data are expressed as means \pm SE. Mean femoral blood flow, mean heart rate and mean arterial blood pressure were calculated from 5-second sub-samples of the data, representing approximately 500 flow and pressure measurements every 15 minutes. Vascular resistance in the hind leg was calculated as mean arterial blood pressure in millimetres of mercury divided by femoral blood flow in millilitres per minute and expressed as resistance units.

Statistical analysis. In order to ascertain differences between treatment groups, repeated-measures ANOVA was used. When 


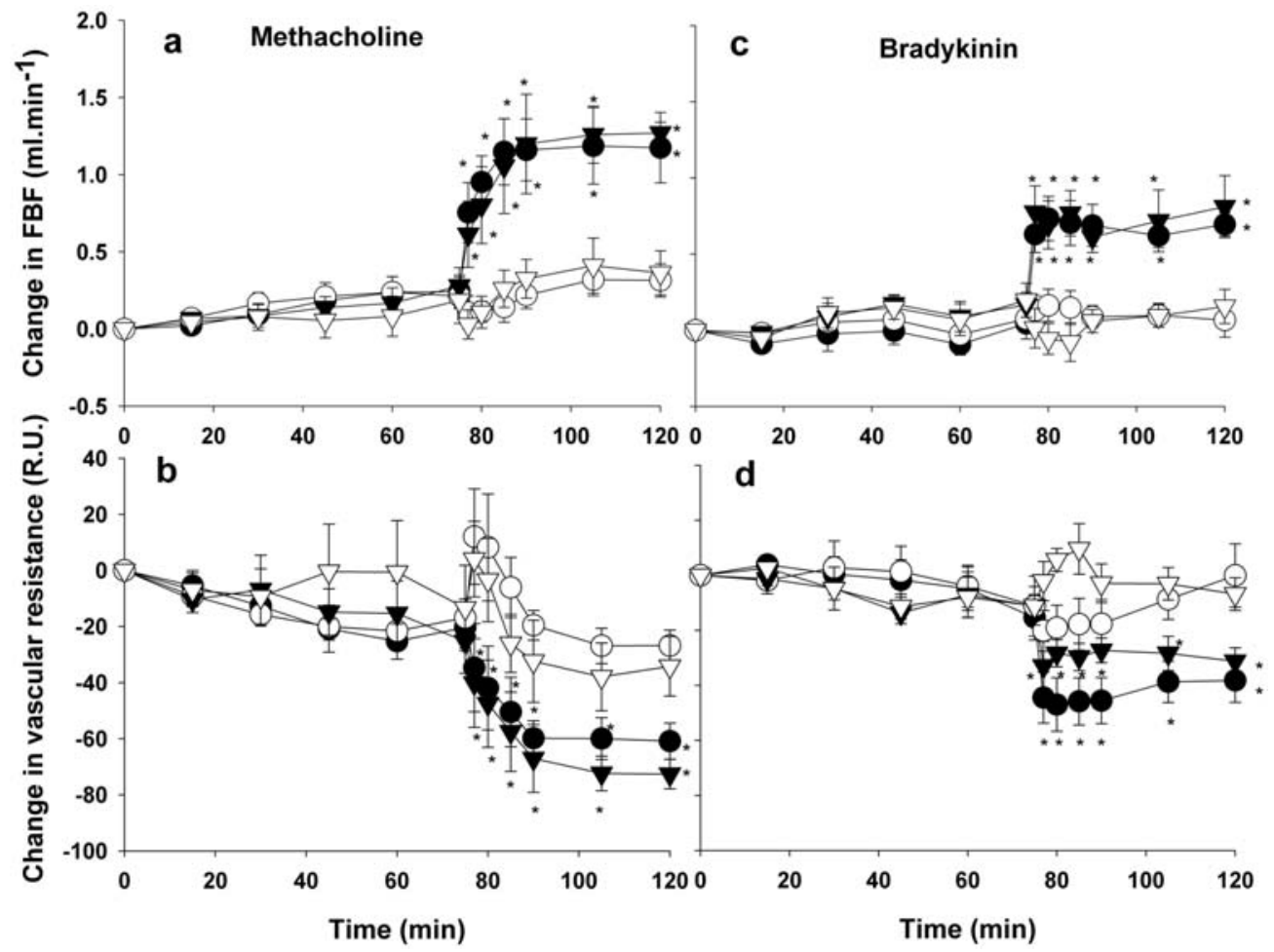

Fig. 2. Change in FBF and VR as a result of local infusion of methacholine $(\mathbf{a}, \mathbf{b})$ or bradykinin $(\mathbf{c}, \mathbf{d})$ via the epigastric artery into one leg with or without systemic infusion of insulin (details, see in Fig. 1). Open circles, control leg saline-treated; filled circles, test leg saline-treated; open triangles, control leg insulin-treated; filled triangles, test leg insulin-treated. $* p<0.05$ for difference from control leg

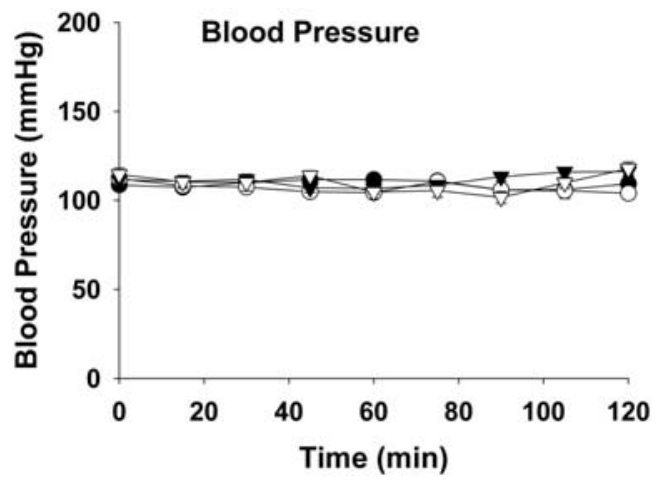

Fig. 3. Effect of local one-leg infusion of methacholine or bradykinin on mean arterial blood pressure. Methacholine or bradykinin were infused via the epigastric artery into one leg with or without systemic infusion of insulin. BP was measured by a cannula in the carotid artery (details, see Fig. 1). Open circles: insulin (control and MC leg); filled circles, saline (control and MC leg); open triangles: insulin (control and BK leg); filled triangle: saline (control and BK leg)

a significant difference $(p<0.05)$ was found, pair-wise comparisons by the Student-Newman-Keuls test were used to determine at which individual time points differences were significant. This was used for femoral blood flow, arterial blood pressure, femoral vascular resistance, and whole-body glucose infusion rate (GIR). For R'g and 1-MX metabolism, one-way
ANOVA was applied, followed by pairwise comparisons using the Student-Newman-Keuls test. All tests were performed using the SigmaStat statistical program (Jandel Software, San Rafael, Calif., USA).

\section{Results}

Haemodynamic effects. Figure 2 shows that values for BP before commencement of methacholine infusion were $110 \pm 3$ (saline) and $112 \pm 2 \mathrm{mmHg}$ (insulin). At 45 min after methacholine infusion there were no significant changes (Fig. 2). Figure 3 shows the change in FBF and vascular resistance (VR) for two different combinations based on details of Figure 1, where methacholine or bradykinin was infused locally via the epigastric artery of the test leg and measurements were made in both legs, while animals were receiving saline infusion systemically or were under hyperinsulinaemic-euglycaemic clamps at $3 \mathrm{mU} \cdot \mathrm{kg}^{-1} \cdot \mathrm{min}^{-1}$. Data for Figure 3 have been normalised relative to the flow at $t=0 \mathrm{~min}$. Thus, absolute values for FBF $\left(\mathrm{ml} \cdot \mathrm{min}^{-1}\right)$ at $t=0 \mathrm{~min}$ were: saline (control leg $1.07 \pm 0.05$; methacholine leg $1.01 \pm 0.02$ ); insulin (control leg 0.88 \pm 0.07 ; methacholine leg $0.91 \pm 0.07$ ); saline (control leg 1.32 \pm 0.08 ; bradykinin leg $1.28 \pm 0.08$ ); and insulin (control leg 1.06 \pm 0.13 ; bradykinin leg $1.19 \pm 0.12$ ).

The rate of local infusion of methacholine was previously determined to maximally increase FBF in the test leg without changes in FBF in the contra-lateral control leg, BP (Fig. 2), or heart rate. Methacholine increased FBF with or without insulin only in the test leg (Fig. 3a) and the concentration attained, as estimated from femoral arterial flow and infusion rate, 


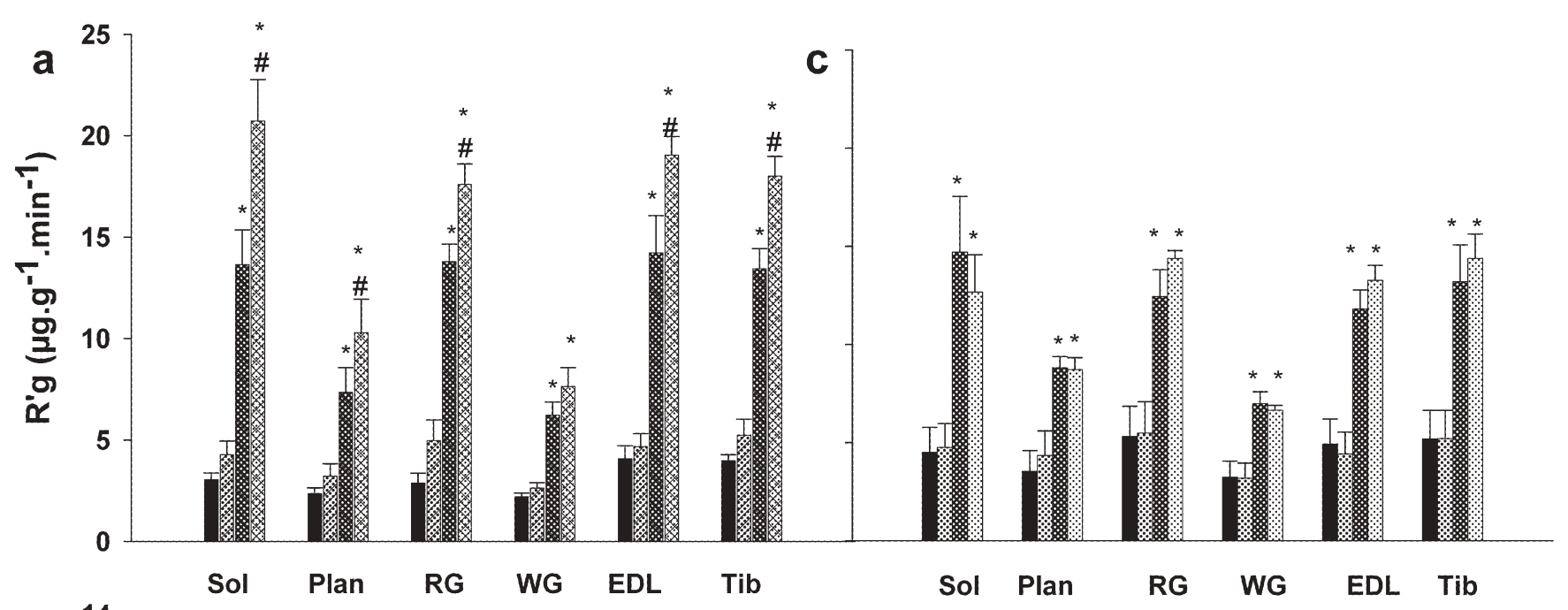

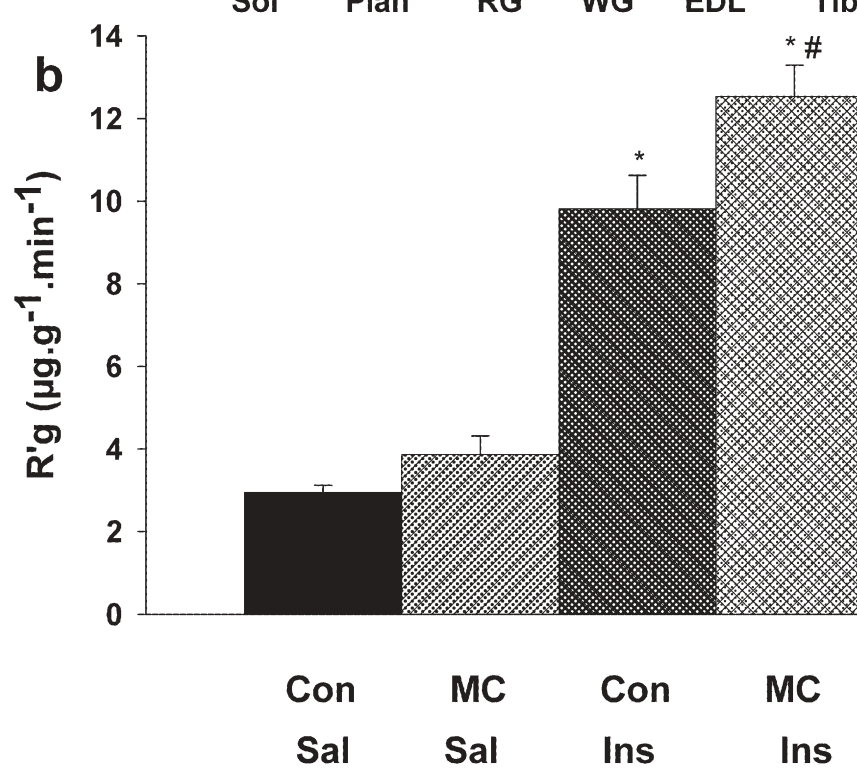

Fig. 4. Effect of local one-leg infusion of methacholine (a, b) or bradykinin $(\mathbf{c}, \mathbf{d})$ on 2-deoxyglucose uptake $\left(\mathrm{R}^{\prime} \mathrm{g}\right)$ by lower leg muscles. R'g for soleus (Sol), plantaris (Plan), red gastrocnemius (RG), white gastrocnemius (WG), extensor digitorum longus (EDL) and tibialis (Tib) muscles are shown (a, c). R'g for the combined muscles above are shown in $\mathbf{b}$ and $\mathbf{d}$. Methacholine or bradykinin was infused in the test leg only; saline or insulin was infused systemically. Control leg saline-treated (con, Sal) test leg saline-treated (MC or BK, Sal) control leg insulin-treated (Con, Ins); test leg insulin-treated (MC or BK, Ins). Other details, see Figure 1. ${ }^{*} p<0.05$ for difference from the corresponding control leg (saline); $\# p<0.001$ for difference from the corresponding control leg (insulin)

was approximately $0.3 \mu \mathrm{mol} \cdot \mathrm{l}^{-1}$. Consequently, the calculated VR in the leg receiving methacholine was found to decrease whether or not insulin was infused (Fig. 3). Bradykinin infusion, like that for methacholine, was also previously tested to produce maximal increase in FBF without systemic effects of changes in BP (Fig. 2) or heart rate (not shown). The concentration attained was estimated to be approximately $0.07 \mu \mathrm{mol} \cdot \mathrm{l}^{-1}$ and the increase in FBF slightly lower than that produced by methacholine; higher infusion

d 

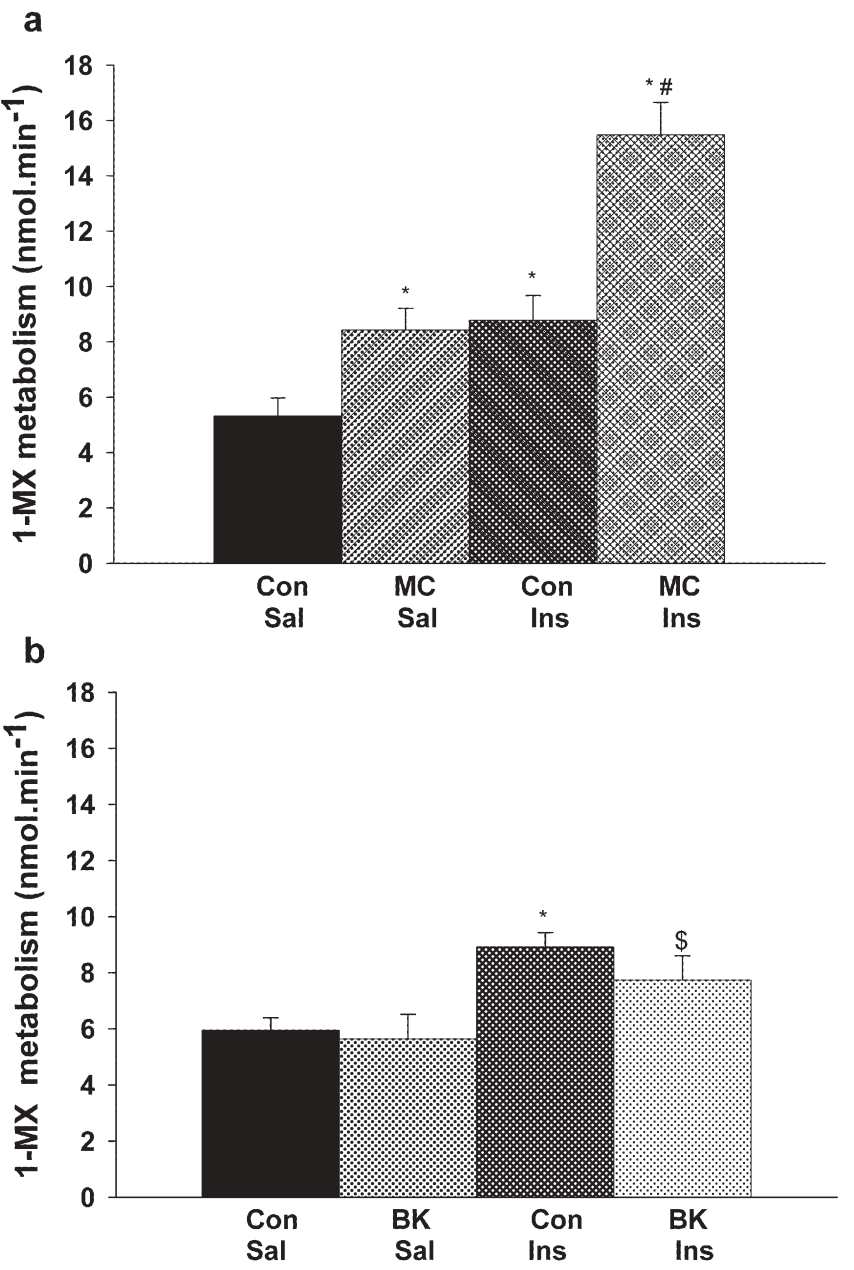

Fig. 5. Effect of local one-leg infusion of methacholine (MC) or bradykinin $(\mathrm{BK})$ on 1-MX metabolism. MC (a) or BK (b) was infused only in the test leg; saline or insulin was infused systemically. From left to right: control leg saline-treated (Con, Sal); test leg saline-treated (MC or BK, Sal); control leg insulin-treated (Con, Ins); test leg insulin-treated (MC or BK, Ins). Other details, see Figure $1 . * p<0.05$ for difference from control leg (saline); \# $p<0.01$ for difference from the corresponding control leg (insulin); $\$ p<0.05$ for difference from test leg without insulin

$5.6 \pm 0.3 \mathrm{mmol} \cdot \mathrm{l}^{-1}$ (insulin). The glucose infusion rate to maintain euglycaemia during insulin reached a plateau at $8.7 \pm 0.9 \mathrm{mg} \cdot \mathrm{kg}^{-1} \cdot \mathrm{min}^{-1}$. For bradykinin the blood glucose values at $0 \mathrm{~min}$ were $4.4 \pm 0.2$ (saline) and $4.0 \pm 0.2 \mathrm{mmol} \cdot \mathrm{l}^{-1}$ (insulin) and at $120 \mathrm{~min} 4.7 \pm 0.2$ (saline) and $4.8 \pm 0.3 \mathrm{mmol} \cdot \mathrm{l}^{-1}$ (insulin). The glucose infusion rate to maintain euglycaemia when clamps were conducted during local bradykinin infusions reached a plateau at $9.4 \pm 0.6 \mathrm{mg} \cdot \mathrm{kg}^{-1} \cdot \mathrm{min}^{-1}$. Local infusions of either methacholine or bradykinin had no effect on systemic plasma insulin. Thus, plasma insulin concentrations at -5 and 120 min were $382 \pm 117$ and $428 \pm 37 \mathrm{pmol} \cdot \mathrm{l}^{-1}$ (methacholine), $325 \pm 134$ and $819 \pm 104 \mathrm{pmol} \cdot \mathrm{l}^{-1}$ (insulin + methacholine), $352 \pm 59$ and $393 \pm 87 \mathrm{pmol} / \mathrm{l}$ (bradykinin), and $312 \pm 51$ and $756 \pm 69 \mathrm{pmol} \cdot \mathrm{l}^{-1}$ (insulin + bradykinin).
Figure 4 shows data for $\mathrm{R}^{\prime} \mathrm{g}$ of individual muscles of the lower leg. Methacholine had no effect on R'g of any of the muscles from the leg into which it was infused. However, methacholine, when infused locally on a background of systemically infused insulin, augmented the insulin-mediated increase in $R^{\prime} g$ for soleus, plantaris, red gastrocnemius, extensor digitorum longus and tibialis muscles (Fig. 4a). For the muscle combination, insulin increased $\mathrm{R}^{\prime} \mathrm{g}$ approximately 3-fold from $3.0 \pm 0.2$ to $9.8 \pm 0.8 \mu \mathrm{g} \cdot \mathrm{g}^{-1} \cdot \mathrm{min}^{-1}$ and this was further increased to $12.5 \pm 0.8 \mu \mathrm{g} \cdot \mathrm{g}^{-1} \cdot \mathrm{min}^{-1}$ by methacholine $(p<0.001) \quad$ (Fig. $4 \mathrm{~b})$. Bradykinin alone (saline background) had no effect on R'g of any of the muscles from the leg into which it was infused (Fig. 4c). Bradykinin (insulin clamp background), unlike methacholine, did not affect the insulin-mediated increase in $\mathrm{R}^{\prime} \mathrm{g}$ of any of the individual muscles (Fig. 4c) or of the combined group (Fig. 4d) of the leg into which it was infused.

Capillary recruitment. Since capillary recruitment is calculated from arterio-venous difference of 1-MX and flow, it is essential for comparisons that the arterial concentrations of 1-MX and the xanthine oxidase inhibitor, oxypurinol, are similar between experimental groups. For the two groups 'saline + local methacholine' and 'insulin + local methacholine' there was no significant difference in either arterial plasma concentrations of $1-\mathrm{MX}\left(16 \pm 1.4\right.$ and $20 \pm 3 \mu \mathrm{mol} \cdot \mathrm{l}^{-1}$ respectively) or oxypurinol $\left(3.5 \pm 0.5\right.$ and $4.0 \pm 1.2 \mu \mathrm{mol} \cdot \mathrm{l}^{-1}$ respectively). Local infusion of methacholine increased capillary recruitment from $5.3 \pm 0.7$ to $8.4 \pm 0.8 \mathrm{nmol} \cdot \mathrm{min}^{-1}$ $(p<0.05)$ in the test leg, as judged from 1-MX metabolism. This increase was significant when compared to the contra-lateral control leg $(p<0.05)$. Systemic insulin infusion also increased 1-MX metabolism. This was significant when control legs were compared $(p<0.05$; Fig. 5a). Further increases in $1-\mathrm{MX}$ from $8.8 \pm 0.9$ to $15.5 \pm 1.2 \mathrm{nmol} \cdot \mathrm{min}^{-1}(p<0.008)$ were seen in the test leg when methacholine was infused locally on a background of insulin clamp (Fig. 5a). Bradykinin alone (saline background) did not increase 1-MX metabolism and did not further modify the stimulation due to systemic insulin infusion (Fig. 5b).

\section{Discussion}

The striking finding from this study was that of the two vasodilators, methacholine and bradykinin, only methacholine enhanced insulin-mediated glucose uptake by muscle in vivo and that this effect of methacholine was associated with a marked augmentation of capillary recruitment. Thus each vasodilator when infused locally into one leg markedly enhanced bulk flow, but despite this increase, only one of these vasodilators enhanced both capillary recruitment and muscle glucose uptake in the presence of physiologi- 
cal insulin. Neither vasodilator was able to increase muscle glucose uptake when infused alone. Thus three important issues emerge from this study. First, it would seem unlikely that bulk flow (FBF) with or without added insulin controls muscle glucose uptake. Second, enhancement of capillary recruitment by methacholine alone without an accompanying increase in glucose uptake would suggest that capillary recruitment alone is not itself a stimulator of glucose uptake (a corollary herein is that methacholine itself is not a stimulator of muscle glucose uptake). Third, augmentation of capillary recruitment and muscle glucose uptake by methacholine, when added with systemic insulin, suggests that capillary recruitment is rate-limiting even for physiological insulin. Thus any intervention that augments capillary recruitment in the presence of elevated insulin (i.e. above basal) will enhance insulin-mediated glucose uptake. Such interventions may include exercise.

There is considerable evidence that the increase in limb blood flow due to insulin is NO-dependent in human subjects $[5,6]$. However, attempts to stimulate muscle glucose uptake by increasing limb blood flow with NO-dependent and -independent vasodilators have been notably unsuccessful. Thus sodium nitroprusside [14], adenosine [15], and bradykinin [13], which markedly increase limb blood flow, do not increase muscle glucose uptake when infused locally in humans [14]. Similarly when epinephrine [4] is infused systemically in rats [4] in vivo, there is no increase in glucose uptake despite increased total flow. In addition, sodium nitroprusside [14] and adenosine [15] fail to ameliorate insulin resistance in insulin-resistant patients, even though many of these patients show a marked loss of NO-dependent insulin- [22] and cholinergic-mediated [23] vasodilation. An exception to the general rule that nitro-vasodilators do not increase muscle glucose uptake appears to be the acetylcholine congener, methacholine. Two laboratories have now reported that this agent increases muscle glucose uptake; in one study it augmented insulin in a range of variably responsive individuals [24], in the other it increased glucose uptake in insulin-resistant hypertensive patients [16], even though nitroprusside in the same patients did not [16]. An explanation for this apparent contradiction may lie in the effect that each vasodilator has on the muscle microvascular blood flow pattern [3]. In rats, insulin and exercise have each been shown to increase capillary recruitment [25], a process that is independent of changes in limb blood flow [11]. Capillary recruitment increases insulin and glucose access to muscle fibres by increasing the proportion of nutritive blood flow. The present study shows that of the two vasodilators, methacholine and bradykinin, only methacholine increases capillary recruitment and augments insulin-mediated glucose uptake. It is also important to note that in the present study there was no difference in FBF when methacholine was infused into the test leg of the rats receiving either systemic insulin or saline. This might imply that insulin and methacholine are not additive, although findings by others [26] suggesting an additive effect between insulin and acetylcholine on human forearm blood flow may indicate otherwise.

The findings reported in the present study have only been possible by the use of a novel procedure in the rat, whereby local infusion of the vasodilator was made via a catheter placed in the epigastric artery of the test leg (Fig. 1a). To our knowledge this approach has not been used before in rats and has allowed assessment of local effects of the vasodilator either on a background of saline or a hyperinsulinaemic-euglycaemic clamp. The particular advantage of this technique is that systemic effects of each vasodilator were kept to a minimum as a result of the infusion being local and the relatively short half-life of the agents. Evidence that systemic effects were minimal can be seen from the unchanged blood pressure and the absence of an increased FBF in the contra-lateral leg. In contrast, systemic infusion of methacholine at a dose that achieved a lower increase in FBF than that from local infusion caused BP to fall and blood glucose to rise, resulting in a lower GIR. Thus systemic effects of methacholine interacted with homeostatic processes and overrode the local metabolic and haemodynamic effects of methacholine in the muscle.

Clearly an explanation as to why methacholine and not bradykinin increased capillary recruitment and glucose uptake must focus on the specific site(s) in the microvasculature at which each substance acts to decrease hindlimb vascular resistance. As already pointed out by others [3], the vasodilatory action of methacholine occurs in large part through release of the endogenous vasodilator NO from arterial endothelial cells [27] and its effects are likely to occur at the level of the high-resistance small precapillary arteries. This is presumably because of the specific location of receptors for methacholine and the local action of the NO released. The failure of bradykinin to achieve the same outcome as methacholine suggests that the receptors for bradykinin are not located at the level of the high-resistance small precapillary arteries, but rather at levels where flow that is essentially non-nutritive can be accommodated. There is already evidence that vasodilators such as bradykinin, acetylcholine, histamine and isoproterenol can depress the contractile force of exercising muscle perfused at constant flow [28]. The authors of that study concluded that the vasodilators diverted blood flow to connective tissue away from the contracting fibres [28]. The fact that acetylcholine was one of the vasodilators that reduced contractile force suggests that despite the close structural properties with methacholine, the latter reacts with a specific subset of receptors controlling nutritive capillary recruitment and is therefore in this respect similar to insulin [4]. 
In the present study methacholine alone increased capillary recruitment with a trend, which may have proved significant with further experiments, to increase $\mathrm{R}^{\prime} \mathrm{g}$ by approximately $29 \%$ or $0.95 \mu \mathrm{g} \cdot \mathrm{g}^{-1} \cdot \mathrm{min}^{-1}$. However, the increment induced by methacholine in the presence of infused insulin, although of the same proportion as that under basal conditions, was larger in absolute terms at $2.7 \mu \mathrm{g} \cdot \mathrm{g}^{-1} \cdot \mathrm{min}^{-1}$. This suggests that capillary recruitment may enhance insulin generally with relatively small effects at basal levels and larger effects when insulin levels rise. This is consistent with our recent data, where we report that capillary recruitment is markedly more sensitive than $\mathrm{R}^{\prime} \mathrm{g}$ to insulin [11]. Thus infusion of physiological insulin at $3 \mathrm{mU} \cdot \mathrm{kg}^{-1} \cdot \mathrm{min}^{-1}$ increased insulin from $337 \pm 31$ to $638 \pm 84 \mathrm{pmol} \cdot \mathrm{l}^{-1}(p<0.05)$ in fed rats and maximally increased capillary recruitment with only about a $20 \%$ of maximal stimulation of $\mathrm{R}^{\prime} \mathrm{g}$ [11]. However, in the present study, when methacholine was infused on a background of the insulin clamp, the increase in $\mathrm{R}^{\prime} \mathrm{g}$ due to insulin was augmented. More importantly, methacholine further increased capillary recruitment due to insulin. These findings suggest that the stimulation of capillary recruitment by methacholine is independent of and additive to that of insulin, but as pointed out above, likely to be engaging receptors in the same locality of the microvasculature. It is perhaps pertinent to note that voluntary exercise-training of our rats also augmented insulin-mediated capillary recruitment and insulin-mediated glucose uptake by muscle [29].

Finally, the present findings separate limb blood flow from muscle glucose uptake. Thus both of the vasodilators increased FBF, but only methacholine augmented insulin-mediated increase in $\mathrm{R}^{\prime} \mathrm{g}$. This to some degree clarifies the on-going controversy where the role of bulk blood flow as an independent modulator of glucose uptake has been disputed [12, 30, 31], particularly in relation to vasodilators [14]. Clearly then, the key haemodynamic aspect that controls insulin-mediated glucose uptake by muscle is capillary surface area. Only vasodilators that act to recruit nutritive capillaries can potentiate insulin action to increase glucose uptake. It remains to be further investigated whether flow can independently increase glucose uptake once capillary recruitment is maximal as predicted elsewhere [32].

Acknowledgements. This work was supported in part by grants from the National Health and Medical Research Council and National Heart Foundation of Australia.

\section{References}

1. Halseth AE, Bracy DP, Wasserman DH (2000) Limitations to basal and insulin-stimulated skeletal muscle glucose uptake in the high-fat-fed rat. Am J Physiol Endocrinol Metab 279:E1064-E1071
2. Sjostrand M, Gudbjornsdottir S, Holmang A, Lonn L, Strindberg L, Lonnroth P (2002) Delayed transcapillary transport of insulin to muscle interstitial fluid in obese subjects. Diabetes 51:2742-2748

3. Baron AD, Steinberg H, Brechtel G, Johnson A (1994) Skeletal muscle blood flow independently modulates insulin-mediated glucose uptake. Am J Physiol 266:E248-E253

4. Rattigan S, Clark MG, Barrett EJ (1997) Hemodynamic actions of insulin in rat skeletal muscle: evidence for capillary recruitment. Diabetes 46:1381-1388

5. Steinberg HO, Brechtel G, Johnson A, Fineberg N, Baron AD (1994) Insulin-mediated skeletal muscle vasodilation is nitric oxide dependent. A novel action of insulin to increase nitric oxide release. J Clin Invest 94:1172-1179

6. Scherrer U, Randin D, Vollenweider P, Vollenweider L, Nicod P (1994) Nitric oxide release accounts for insulin's vascular effects in humans. J Clin Invest 94:2511-2515

7. Vincent MA, Barrett EJ, Lindner JR, Clark MG, Rattigan S (2003) Inhibiting NOS blocks microvascular recruitment and blunts glucose uptake in response to insulin. Am J Physiol 285:E123-E129

8. Clark MG, Wallis MG, Barrett EJ et al. (2003) Blood flow and muscle metabolism: a focus on insulin action. Am J Physiol Endocrinol Metab 284:E241-E258

9. Rattigan S, Clark MG, Barrett EJ (1999) Acute vasoconstriction-induced insulin resistance in rat muscle in vivo. Diabetes 48:564-569

10. Vincent MA, Barrett EJ (2002) Insulin-induced capillary recruitment precedes changes in skeletal muscle glucose uptake. Diabetes 51 [Suppl 2]:A31

11. Zhang L, Vincent MA, Richards SM et al. (2004) Insulin sensitivity of muscle capillary recruitment in vivo. Diabetes 53:447-453

12. Yki-Jarvinen H, Utriainen T (1998) Insulin-induced vasodilatation: physiology or pharmacology? Diabetologia 41: 369-379

13. Nuutila P, Raitakari M, Laine H et al. (1996) Role of blood flow in regulating insulin-stimulated glucose uptake in humans. Studies using bradykinin, $\left[{ }^{15} \mathrm{O}\right]$ water, and $\left[{ }^{18} \mathrm{~F}\right]$ fluoro-deoxy-glucose and positron emission tomography. J Clin Invest 97:1741-1747

14. Natali A, Quinones GA, Pecori N, Sanna G, Toschi E, Ferrannini E (1998) Vasodilation with sodium nitroprusside does not improve insulin action in essential hypertension. Hypertension 31:632-636

15. Natali A, Bonadonna R, Santoro D et al. (1994) Insulin resistance and vasodilation in essential hypertension. Studies with adenosine. J Clin Invest 94:1570-1576

16. Sarabi M, Lind L, Millgard J et al. (1999) Local vasodilatation with methacholine, but not with nitroprusside, increases forearm glucose uptake. Physiol Res 48:291-295

17. Jarasch ED, Bruder G, Heid HW (1986) Significance of xanthine oxidase in capillary endothelial cells. Acta Physiol Scand 548 [Suppl]:39-46

18. Emmerson BT, Gordon RB, Cross M, Thomson DB (1987) Plasma oxipurinol concentrations during allopurinol therapy. Br J Rheumatol 26:445-449

19. Rattigan S, Appleby GJ, Miller KA et al. (1997) Serotonin inhibition of 1-methylxanthine metabolism parallels its vasoconstrictor activity and inhibition of oxygen uptake in perfused rat hindlimb. Acta Physiol Scand 161:161-169

20. Kraegen EW, James DE, Jenkins AB, Chisholm DJ (1985) Dose-response curves for in vivo insulin sensitivity in individual tissues in rats. Am J Physiol 248:E353-E362

21. James DE, Jenkins AB, Kraegen EW (1985) Heterogeneity of insulin action in individual muscles in vivo: euglycemic clamp studies in rats. Am J Physiol 248:E567-E574 
22. Laakso M, Edelman SV, Brechtel G, Baron AD (1992) Impaired insulin-mediated skeletal muscle blood flow in patients with NIDDM. Diabetes 41:1076-1083

23. Serne EH, Gans RO, ter Maaten JC, ter Wee PM, Donker AJ, Stehouwer CD (2001) Capillary recruitment is impaired in essential hypertension and relates to insulin's metabolic and vascular actions. Cardiovasc Res 49:161168

24. Baron AD, Tarshoby M, Hook G et al. (2000) Interaction between insulin sensitivity and muscle perfusion on glucose uptake in human skeletal muscle: evidence for capillary recruitment. Diabetes 49:768-774

25. Dawson D, Vincent MA, Barrett EJ et al. (2002) Vascular recruitment in skeletal muscle during exercise and hyperinsulinemia assessed by contrast ultrasound. Am J Physiol Endocrinol Metab 282:E714-E720

26. Rask-Madsen C, Ihlemann N, Krarup T et al. (2001) Insulin therapy improves insulin-stimulated endothelial function in patients with type 2 diabetes and ischemic heart disease. Diabetes 50:2611-2618
27. Vallance P, Collier J, Moncada S (1989) Effects of endothelium-derived nitric oxide on peripheral arteriolar tone in man. Lancet 2:997-1000

28. Hirvonen L, Korobkin M, Sonnenschein RR, Wright DL (1964) Depression of contractile force of skeletal muscle by intra-arterial vasodilator drugs. Circ Res 14:525-535

29. Rattigan S, Wallis MG, Youd JM, Clark MG (2001) Exercise training improves insulin-mediated capillary recruitment in association with glucose uptake in rat hind limb. Diabetes 50:2659-2665

30. Steinberg HO, Baron AD (1999) Insulin-mediated vasodilation: why one's physiology could be the other's pharmacology. Diabetologia 42:493-495

31. Natali A (1997) Skeletal muscle blood flow and insulin action. Nutr Metab Cardiovasc Dis 7:105-109

32. Clerk LH, Vincent MA, Lindner JR, Clark MG, Rattigan S, Barrett EJ (2004) The vasodilatory actions of insulin on resistance and terminal arterioles and their impact on muscle glucose uptake. Diabetes Metab Res Rev 20:3-12 